\title{
Comparison of Aspiration Catheters with Modified Standard Catheters for Treatment of Large Pulmonary Embolism Using an In-vitro Patho-Physiological Model
}

\author{
Franziska Schubert $^{1}\left(\mathbb{D} \cdot\right.$ Masashi Tamura $^{2,3} \cdot$ Sophie Bezela $^{1} \cdot$ Alexander Weyers $^{1}$. \\ Daniel Kütting ${ }^{4} \cdot$ Matthias Menne $^{1} \cdot$ Ulrich Steinseifer $^{1} \cdot$ Johanna C. Clauser $^{1}$. \\ Thomas Schmitz-Rode ${ }^{3}$
}

Received: 27 April 2021 / Accepted: 6 October 2021 / Published online: 18 November 2021

(C) The Author(s) 2021

\begin{abstract}
Purpose The presented in-vitro study provides a comparison of various catheters for mechanical treatment of largeburden pulmonary embolism (PE) under standardized conditions, using a new test rig. Dedicated aspiration catheters (JETi $\AA$, Penumbra Indigo $\AA$, Aspirex $\AA$ ) were compared with standard catheters (Pigtail, Multi-Purpose, Balloon Catheter) applied for embolus fragmentation.

Materials and Methods Emboli prepared from porcine blood were washed into the test rig which consists of anatomical models of the pulmonary artery (PA) and of the right heart in combination with a pulsatile drive system. For all catheters, the duration of the recanalization procedure and the weight percentage (wt\%) of the remaining, removed and washed-down clot fractions were evaluated. For aspiration catheters, the aspirated volume was measured.

Results All catheters achieved full or partial recanalization. The aspiration catheters showed a significantly $(\mathrm{p}<0.05)$ lower procedure time $(3: 15 \mathrm{~min} \pm 4: 26 \mathrm{~min})$ than the standard fragmentation catheters $(7: 19 \mathrm{~min} \pm$
\end{abstract}

Franziska Schubert

neukamp@ame.rwth-aachen.de

1 Department of Cardiovascular Engineering, Institute of Applied Medical Engineering, Helmholtz Institute, RWTH Aachen University and University Hospital, Aachen, Germany

2 Department of Radiology, Keio University School of Medicine, Tokyo, Japan

3 Institute of Applied Medical Engineering, Helmholtz Institute, RWTH Aachen University and University Hospital, Aachen, Germany

4 Department of Radiology, University of Bonn, Bonn, Germany
4:40 min). The amount of thrombus removed by aspiration was significantly $(\mathrm{p}<0.001)$ higher than that by fragmentation, averaging $86.1 \mathrm{wt} \% \pm 15.6 \mathrm{wt} \%$ and 31.7 $\mathrm{wt} \% \pm 3.8 \mathrm{wt} \%$, respectively. Nonetheless, most of the residue was fragmented into pieces of $\geq 1 \mathrm{~mm}$ and washed down. Only in 2 of 36 tests, a residual thrombus of $11.9 \mathrm{wt} \% \pm 5.1 \mathrm{wt} \%$ remained in the central PA.

Conclusion Comparison under standardized in-vitro pathophysiological conditions showed that embolus fragmentation with standard catheters is clearly inferior to aspiration with dedicated catheters in the treatment of large-burden PE, but can still achieve considerable success.

Level of Evidence No level of evidence, experimental study.

Keywords Pulmonary embolism · Interventional radiology · Mechanical thrombectomy ·

Fragmentation · Aspiration · Comparative in-vitro study $\cdot$ Patho-physiological model

\section{Introduction}

Although a decrease in mortality related to pulmonary embolism (PE) is indicated by the WHO Mortality Database, PE causes an average of 6.5 deaths per 100,000 population annually and remains a serious clinical issue [1-3]. In particular, COVID-19 is reported to potentially promote $\mathrm{PE}$ resulting in sudden death even in young patients [4-6].

For patients with acute large-burden PE who cannot be treated by either systemic thrombolysis or surgical 
intervention, "catheter-directed therapy" is a promising approach [7-11]. It comprises mechanical thrombectomy including mechanical extraction and fragmentation of the thrombus as well as (adjunctive) catheter-mediated thrombolytic therapy [11]. Among mechanical devices, several catheters have been developed and tested over the past decades [10-12]. Standard pigtail or multi-purpose catheters have been introduced for emergency mechanical fragmentation of PE [13]. A slight modification of the pigtail catheter enabled additional rotation [14, 15]. Moreover, technically more complex solutions with aspiration were assessed for the treatment of $\mathrm{PE}$, like the Aspirex ${ }^{\circledR}$ System (Straub Medical AG, Wangs, Switzerland) or the Penumbra Indigo ${ }^{\circledR}$ System (Penumbra, Inc., Alameda, CA, USA) [16, 17]. Large-bore devices that use both mechanical and aspiration mechanisms to remove the clot, such as the FlowTriever ${ }^{\circledR}$ Retrieval/Aspiration System (Inari Medical, Irvine, California), also show promising results [18]. However, the performance of such dedicated aspiration devices has not yet been compared to that of standard catheters.

The main objective of this in-vitro study is to compare dedicated aspiration catheters with slightly modified conventional catheters for fragmentation in the treatment of large PE. The term "large PE" is used in the sense of a large thrombus burden with pulmonary arterial pressure elevation. Since a valid comparison requires a standardized environment, a new test rig is proposed that provides near patho- physiological and anatomical conditions.

\section{Materials and Methods}

\section{Experimental Setup}

For clot preparation, porcine blood was sampled under exsanguination by cutting the carotid artery in an abattoir setting. The permission to use porcine blood was granted by the responsible veterinary authority in accordance with Article 23 of Regulation (EC) No 1069/2009. For consolidation purposes, the blood was shelved for one week in a refrigerator at $4{ }^{\circ} \mathrm{C}$. On the day of measurement, single cylindrical clots with a length of $100 \mathrm{~mm}$, a diameter of approx. $15 \mathrm{~mm}$ and an average weight of $6.54 \mathrm{~g} \pm 0.15 \mathrm{~g}$ were stamped from the solidified block of clotted blood, serving in the model as thrombus with increased consistency compared to fresh thrombus.

A novel test rig was designed for this study to achieve anatomical and patho-physiological in-vitro conditions. The key components of the test rig (Fig. 1) are a glass model of the pulmonary artery (PA) with segmental arteries based on anatomical casts from a human corpse [19] and a transparent 3D-printed (VeroClear, Stratasys
Ltd. ( ), Eden Prairie, MN, USA) right heart model based on CT data. The PA model was adjusted to the right heart, mimicking the pulmonary trunk and enabling an anatomical path for the catheter. The prepared clot was washed either into the right or the left main PA. As the right heart model is rigid, the pump function had to be decoupled. Therefore, the total artificial heart ReinHeart (ReinHeart TAH GmbH, Aachen, Germany) [20] was used as a pump providing a physiological pulsatile flow of $5 \mathrm{~L} / \mathrm{min}$ at $60 \mathrm{bpm}$ [15]. Water at room temperature was used as fluid.

The pulmonary flow $Q_{P}$ was measured using an ultrasound clamp-on tubing sensor (ME20PXL215/TS410, Transonic Systems Inc., Ithaca, NY, USA). The mean pulmonary artery pressure (mPAP) was monitored (Xtrans ${ }^{\circledR}$, Codan Medizinische Geräte GmbH \& Co KG, Lensahn, Germany) in the pulmonary trunk of the model. The outflow of all PA branches merged into one single tube leading to a reservoir system consisting of three closed cylindrical tank reservoirs connected in series. Clot fragments from the aspiration or fragmentation procedures were washed downstream and settled on the bottom of the first reservoir. The second reservoir functioned redundantly, catching remaining clot parts that passed the upstream reservoir. The third reservoir was attached to the pump, thus closing the loop. After the procedure, the collected clot fragments from the first two reservoirs were filtered through a set of sieves (Haver\&Boecker OHG, Oelde, Germany) with filter sizes of $5 \mathrm{~mm}, 2 \mathrm{~mm}$ and $1 \mathrm{~mm}$.

PA flow was adjusted by means of a throttle valve on a shunt bypassing the PA and the right heart model. The flow $\mathrm{Q}_{\mathrm{P}}$ was set to $3.5 \mathrm{~L} / \mathrm{min}$, simulating a reduction in cardiac output caused by large thrombus burden PE.

\section{Experimental Interventions}

Two physicians with general experience in interventional radiology-including clinical experience in PE catheter therapy-performed all experiments. Since the test rig allowed direct sight, the procedures were conducted without the use of fluoroscopy. To mimic a jugular approach as closely as possible, the catheter was introduced at the location depicted in Fig. 1. An access sheath with a hemostasis valve was used. For placement of the catheters, similar guiding sheathes (Destination Fr. 8, Ref.: 54-86501, Terumo Medical Corp., Elkton, MD, USA) and guide wires (Radifocus ${ }^{\circledR}$ ， RF-PA35153M/RF-GA35223M， Terumo; TCMTNA-35-145-3, Cook Inc., Bloomington, IN, USA) were applied unless special guide wires were provided by the catheter's manufacturer. In Fig. 2, an example of a test embolus placed within the test setup (left) and the tested catheters (middle and right) are depicted. 


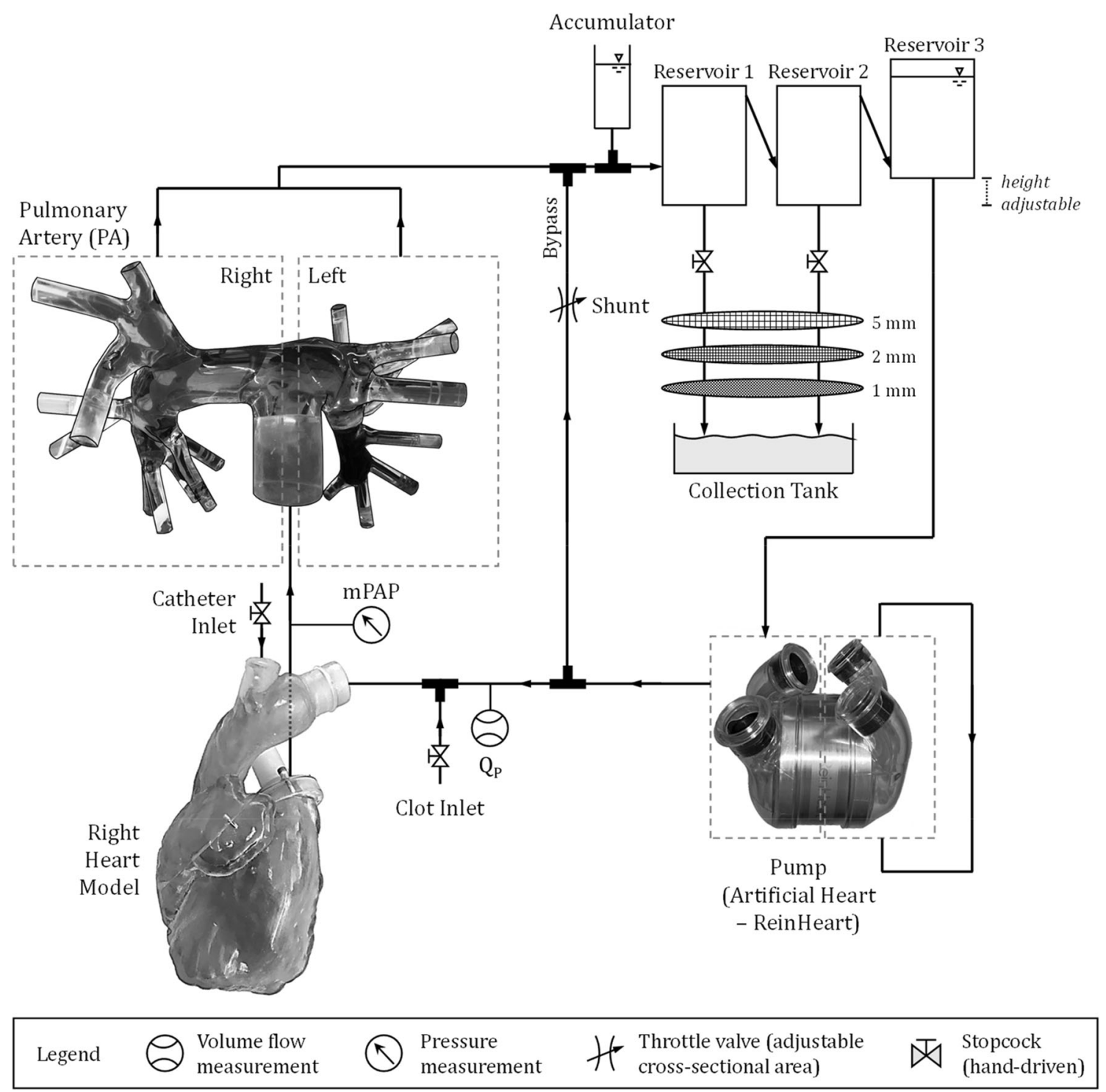

Fig. 1 Schematic illustration of the test rig (dimensions are not representative)

Fig. 2 Clot captured on the right side of the pulmonary artery model (PA) (left); Investigated catheters (middle and right): $\mathrm{PIG}-$

Rotatable Pigtail Catheter, MPY_Rotatable Multi-Purpose Catheter, PTA-Balloon Catheter, JET_JETi ${ }^{\circledR}$ Thrombectomy System, PENPenumbra Indigo ${ }^{\circledR}$ System, ASP_Aspirex ${ }^{\circledR}$
Standard Catheters

Captured Clot

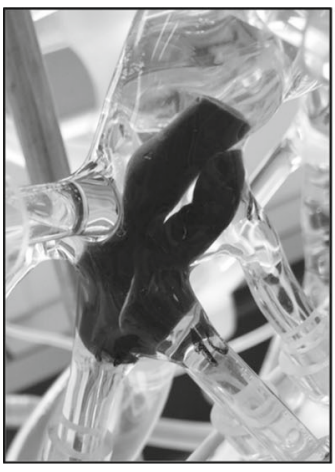

Dedicated Aspiration Catheters
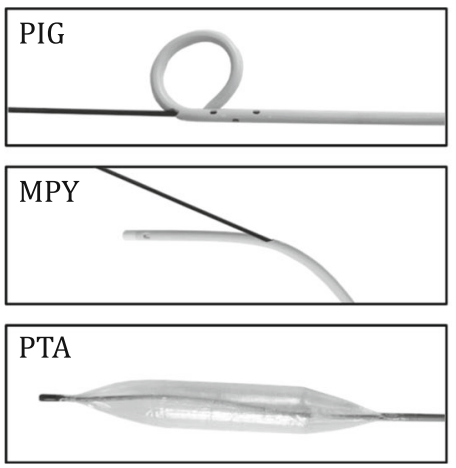
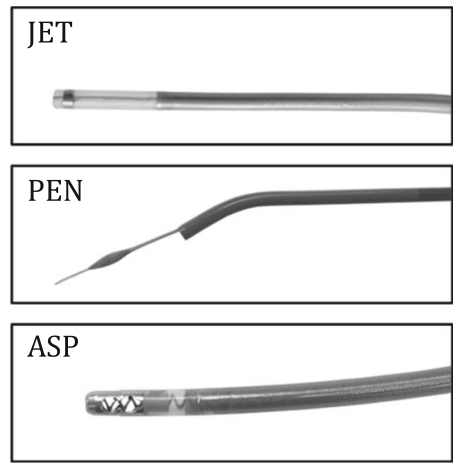


\section{Standard Catheters for Fragmentation}

With the standard catheters shown in Fig. 2 (middle row), fragmentation of the thrombus is performed either by rotation (PIG, MPY) or by repeated compression of the clot (PTA). The respective catheters and, if applicable, their modification are described below.

PIG-Rotatable Pigtail Catheter A commercially available high-torque $145^{\circ}$-angled pigtail catheter with a diameter of $6 \mathrm{~F}$ and a length of $110 \mathrm{~cm}$ (Super Torque ${ }^{\circledR}$ Plus, Ref.: 533-652S, Cordis Corporation, Miami Lakes, FL, USA) was modified as proposed in previous studies [15]. An oval opening (approx. $1 \times 2 \mathrm{~mm}$ ) was cut in the outer curvature of the pigtail loop in straight projection to the catheter shaft (Fig. 2-PIG). In this way, a rotation around the axis of an inserted guide wire protruding out of the oval opening can be performed by manually rotating the proximal catheter shaft. With the pigtail tip placed in an embolus, rotation and simultaneous back-and-forth movement of the catheter achieve a fragmentation [14]. The tip of the guiding sheath was placed within the pulmonary trunk in order to reduce rotation-induced friction.

MPY-Rotatable Multi-Purpose Catheter A commercially available high-torque (Super Torque ${ }^{\circledR}$ Plus) openend multi-purpose catheter with a diameter of $6 \mathrm{~F}$ and a length of $100 \mathrm{~cm}$ (MPA2SH100cm, Ref.: 533-642, Cordis) was modified. An oval opening (approx. $1 \times 2 \mathrm{~mm}$ ) was cut in the transition between outer curvature of the deflected tip portion and the straight catheter shaft (Fig. 2MPY). Similar to PIG, the MPY was used in conjunction with an inserted guide wire and the sheath's tip was placed in the pulmonary trunk.

PTA-Balloon Catheter Placed within an embolus, repeated inflation and deflation of the balloon results in coarse fragmentation of the clot as it is pressed against the vessel's wall. A PTA balloon catheter (Fig. 2-PTA) with a balloon diameter of $14 \mathrm{~mm}$, balloon length of $40 \mathrm{~mm}$, shaft diameter of $6.5 \mathrm{~F}$ and a total length of $100 \mathrm{~mm}$ was selected (Zelos, Ref.: 740-1404, Optimed GmbH, Ettlingen, Germany). Based on preliminary tests with different balloon diameters and lengths, this geometry was chosen as a compromise between maneuverability and fragmentation capability.

\section{Dedicated Aspiration Catheters}

The dedicated devices examined in this study (Fig. 2, right row) use a combination of fragmentation and aspiration to remove the thrombus.

JET-JETi ${ }^{\circledR}$ Thrombectomy System The JETi ${ }^{\circledR}-8$ System (Walk Vascular, LLC., Irvine, CA, USA) with a diameter of $8 \mathrm{~F}$ employs an interior high-pressure jet stream (saline solution) to break up the clot and to remove the crushed material (Fig. 2-JET). The jet is positioned inside the catheter lumen, enabling for clot fragmentation and simultaneous aspiration.

PEN_Penumbra Indigo ${ }^{\circledR}$ System The Penumbra Indigo ${ }^{\circledR}$ System (Penumbra, Inc., Alameda, CA, USA) causes mechanical clot fragmentation using a separator and subsequent suction-based extraction of the clot parts driven by a vacuum pump. In this study, a corresponding system (Fig. 2-PEN) combining an 8F aspiration catheter, a separator CAT8/SEP8 (8F, $115 \mathrm{~cm}$, Ref.: CAT8XTORQ115) and an aspiration tubing (IST3) was evaluated.

ASP-Aspirex ${ }^{\circledR}$ The Aspirex ${ }^{\circledR}$ System (Becton Dickinson, formerly Straub Medical, Wangs, Switzerland) aspirates the clot, shreds the aspirated material and removes it from the patient's body by suction. In the test rig, the 10F Aspirex ${ }^{\circledR}(\mathrm{S} 10 \mathrm{~F}$, Ref.: 80231) and its special guide wire (0.018", 4/220 cm, angled, Ref.: 80233) were examined (Fig. 2-ASP).

\section{Measurements}

Endpoint of the study was the achievement of a complete recanalization of the central PA occlusion. The respective time was measured. In one case treatment was terminated after $20 \mathrm{~min}$. Prior to each experiment, the clot was weighted. Following each procedure, reservoirs 1 and 2 were discharged and the clot residue captured in the sieves was evaluated for its weight (washed-down and filtered clot $\geq 1 \mathrm{~mm}$ ). The fragments' weights were summed up regarding each filter size $(5 \mathrm{~mm}, 2 \mathrm{~mm}$ and $1 \mathrm{~mm})$. Remaining clots in the PA were subsequently removed and weighted. Additionally, the weight of the removed (aspirated or fragmented) clot parts was calculated by subtracting the weight of all washed-down and remaining clot fragments from the embolus' initial weight. For standard catheters, a washed-down clot residue with a size smaller than $1 \mathrm{~mm}$, thus passing all three sieves, was considered as removed as it was no longer detectable. The respective weight percentage (wt \%) referenced to the initial embolus was calculated. In the case of the aspiration catheters, the aspirated fluid volume was also measured.

\section{Statistical Analysis}

Each catheter was tested six times (three times on each PA side). Data analysis was performed using MATLAB ${ }^{\circledR}$ (The MathWorks, Inc., Natick, MA, USA), Version R2017b. For statistical analysis, mean values and standard deviations (SD) were considered. One-way ANOVA tests were carried out using IBM ${ }^{\circledR}$ SPSS ${ }^{\circledR}$ Statistics 24 (IBM Corp. $\odot$, Armonk, NY, USA). In the case of homogeneity of variances, post hoc tests for multiple comparison were performed using Tukey test. If variances proved 
inhomogeneous, Games-Howell post hoc test was applied. To assess significance between the dedicated and standard devices, single factorial ANOVA or Welch tests were performed, depending on the homogeneity of variances. Results were considered significant with $\mathrm{p}<0.05$.

\section{Results}

The average time required to perform each procedure is depicted in Fig. 3. The limit of 20 min was exceeded only once using the MPY. Furthermore, one MPY catheter was damaged at the external shaft portion due to excessive torsion. Both tests are excluded from the calculation. Averaging all tests per catheter type shows a significantly lower procedure time for dedicated devices (3:15 $\mathrm{min} \pm$ 4:26 min) compared to standard catheters $(7: 19 \mathrm{~min} \pm$ 4:40 min). Notably, the most rapid thrombectomy was achieved using JET. Considering only dedicated devices, ASP led to the longest procedures with a significant $(\mathrm{p}<0.01)$ deviation compared to JET. The overall maximum duration for a successful procedure was required when using the PTA device. Among standard catheters,

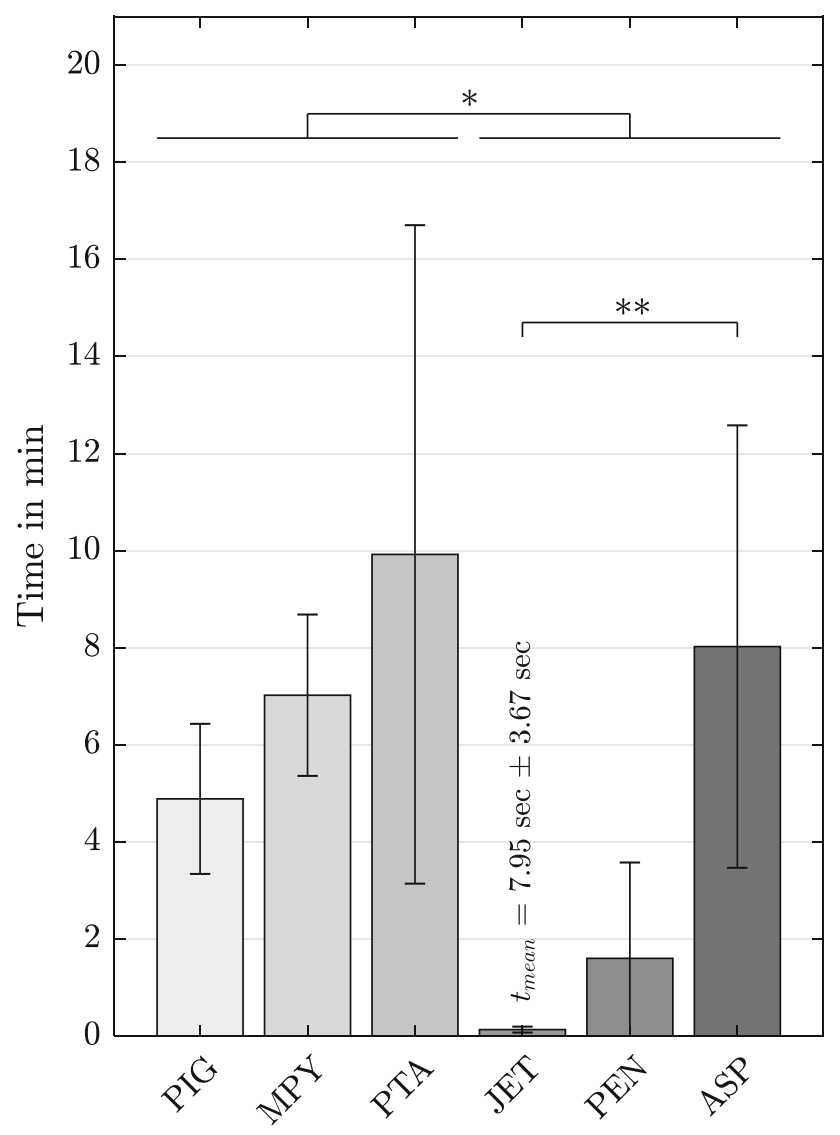

Fig. 3 Duration of the procedure in min (mean, SD) for each catheter $(\mathrm{n}=6$, MPY: $\mathrm{n}=4), * \mathrm{p}<0.05, * * \mathrm{p}<0.005$
PIG showed the shortest operation times, although no significance could be proven in comparison to the other standard devices.

Figure 4 explains the division into thrombus fractions after aspiration or pure fragmentation. Correspondingly, Fig. 5 presents the weight percentage (wt\%) of (1) the remaining, (2) the washed-down and filtered, and (3) the removed clots. As the weight of removed clot fractions is calculated from remaining and filtered fractions, no SDs are specified within the figure. All dedicated thrombectomy devices showed a significantly $(\mathrm{p}<0.001)$ higher amount of thrombus removed by aspiration $(86.1 \mathrm{wt} \% \pm 15.6$ $\mathrm{wt} \%)$ than by fragmentation with standard catheters (31.7 $\mathrm{wt} \% \pm 3.8 \mathrm{wt} \%)$. The JET device allowed an almost complete thrombus aspiration. The two unsuccessful trials with the MPY device led to thrombus fragments remaining in the central PA model. However, the average percentage of the corresponding residual fragments accounted for only $3.97 \mathrm{wt} \% \pm 6.5 \mathrm{wt} \%$ of the initial thrombus (averaged over all six tests). Regarding the washed-down and filtered clot parts, the dedicated aspiration devices led to significantly (JET, PEN: $p<0.001$, ASP: $p<0.05$ ) smaller clot fractions for diameters of $\geq 5 \mathrm{~mm}$ than did the standard catheters (Fig. 6). However, as indicated by the high SDs, no significant differences were observed among all of the devices for clot fractions of smaller diameters $(\geq 2 \mathrm{~mm}, \geq 1 \mathrm{~mm}$ ).

Among the dedicated aspiration devices, the ASP catheter showed a significantly higher fluid loss during aspiration $(317.5 \mathrm{~mL} \pm 205.5 \mathrm{~mL}$ ), while the JET device yielded the highest fluid loss per time $(452.5 \mathrm{~mL} / \mathrm{min} \pm$ $202.3 \mathrm{~mL} / \mathrm{min})$.

\section{Discussion}

In scope of the presented tests, the dedicated thrombus aspiration catheters led to significantly better results in terms of the duration of the procedure and the amount of removed clots. While use of the JET device led to the most effective thrombectomy, considerably high aspiration rates were observed. In a clinical setting, lower aspiration rates might be advantageous, since serious blood loss can result if the active tip of the device is not embedded properly inside the embolus [11, 12]. Although less effective, standard catheters with a simple customization proved useful for fragmentation of large thrombus burden PE by achieving full or at least partial recanalization of the PA.

Considering their handling, PIG and MPY revealed limitations as their size (pigtail curve, deflection curvature) made fragmentation within model PA branches of smaller diameter difficult. Similar results have been reported in a clinical study [21]. Also the generation of larger thrombus 
Fig. 4 Respective fractions of the embolus in relation to its initial weight (percent by weight $\mathrm{wt} \%)$ and example images of the individual washed-down and filtered clots divided by size; for fragmentation with standard catheters, a washed-down clot residue with a size smaller than $1 \mathrm{~mm}$ (thus passing all three sieves) was considered as removed as it was no longer detectable

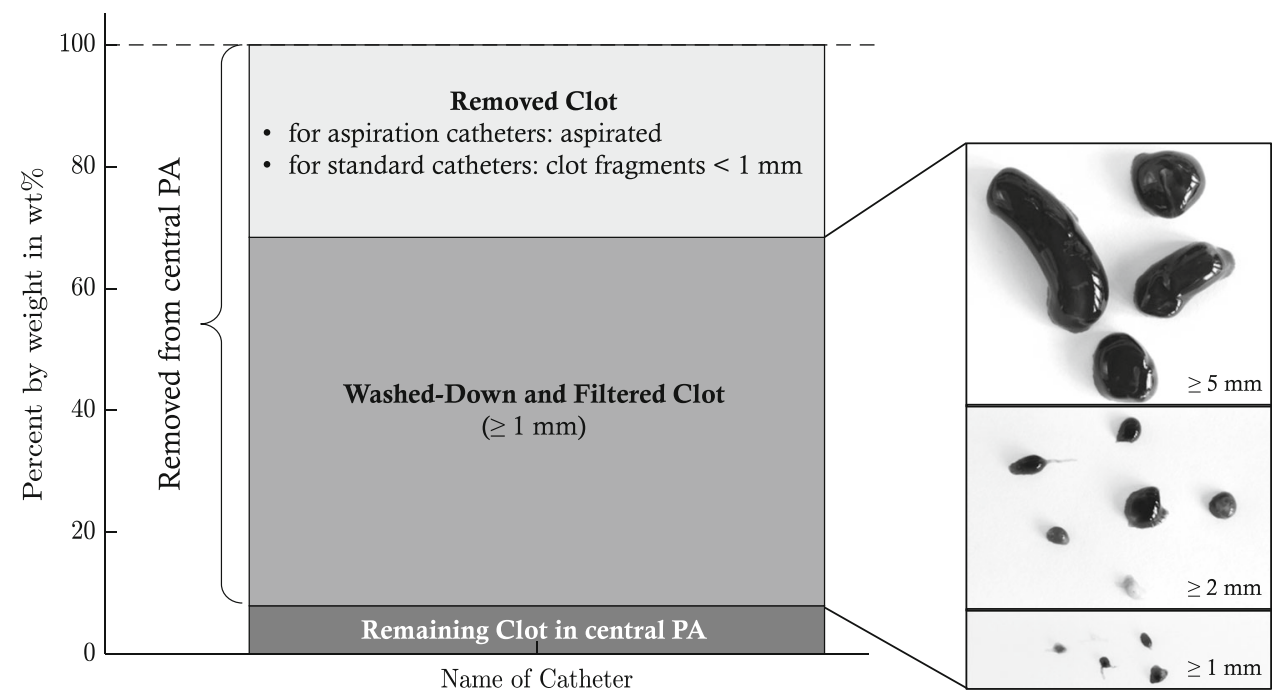

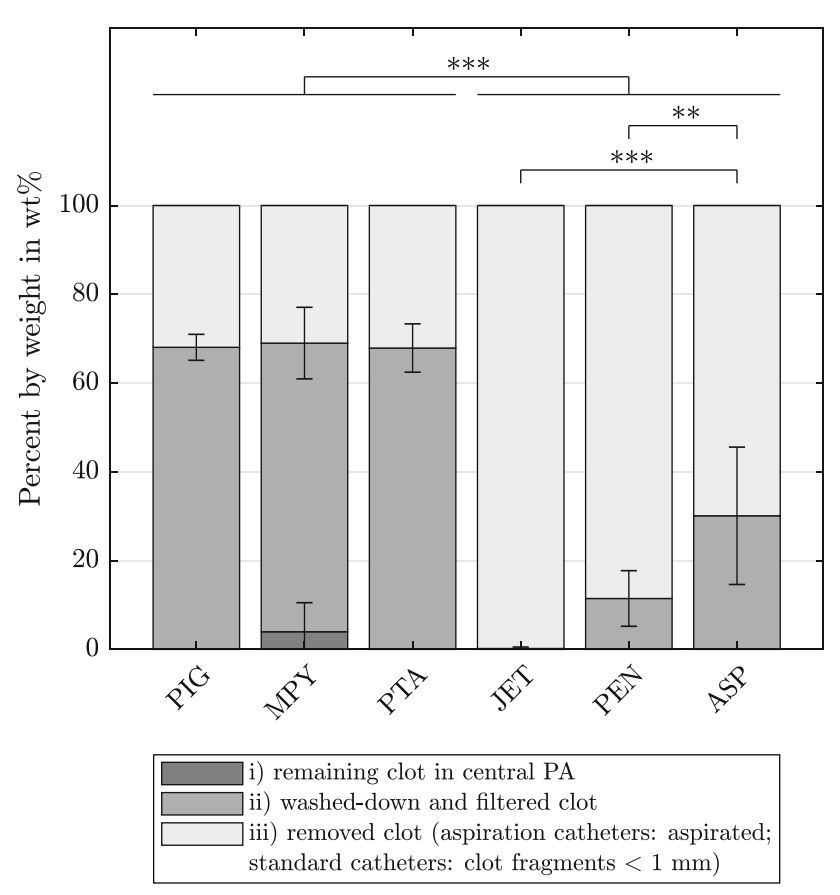

Fig. 5 Amount of remaining clot in central PA, washed-down and filtered clot, and removed clot (aspiration catheters: aspirated; standard catheters: clot fragments $<1 \mathrm{~mm}$ ) related to the initial clot weight in percent by weight wt $\%$ (mean, SD, per respective fraction) for each catheter, $* * \mathrm{p}<0.005, * * * \mathrm{p}<0.001$

fragments capable of obstructing pulmonary branches is consistent with clinical observation [14, 21].

The new test rig offers an increased level of anatomical and patho-physiological resemblance, allowing to generate PE by washing in consistent blood clots and to adjust near patho- physiological pressure and pulsatile flow values. Thus, our setup can simulate clinically observed phenomena that increase procedure time, such as difficult catheter positioning maneuvers, repositioning due to catheter dislocation, dislocation of embolus fragments, and treatment of embolus parts that moved to distal sections. The anatomical detailing of our model differs from that of published setups, for instance the investigation of the thrombectomy properties of the Straub Rotarex ${ }^{\circledR}$ Catheter (Straub Medical, Wangs, Switzerland) used a simple model with straight tubes and a straight access [22]. In the MüLLER-HÜLSBECK model that has been used in different studies [23-25], a thrombus is cultivated inside a straight silicone tube to mimic PA embolism. With this simplified model, low intervention times have been reported for different catheters, e.g., for the Amplatz Thrombectomy Device [23], that do not correlate with clinical reports [26].

\section{Study Limitations}

All procedures were performed under direct visual control. However, as this simplified framework applied equally to all catheters, it should not have significantly affected the comparison. As an improvement of the setup, the implementation of sight restriction in combination with x-ray fluoroscopy could be useful in future trials. Furthermore, the components of the test rig representing the PA and the right heart did not account for ventricle contraction, physiological vessel compliance or vasoconstriction. Also, the procedure-related risk of wall injury could not be evaluated. Furthermore, wall friction is artificial and may influence catheter handling. In future, it could be expedient to fabricate the anatomical structures out of elastic polymer to account for compliance. Moreover, a right heart model with a pumping function by external compression of an elastic ventricle [27] would eliminate the need for an additional pumping device. Because of the test rig's volume (and the need to refill after each trial), water was selected as flow medium. Since pure water impairs the 
Fig. 6 Filtered clot weights in percent by weight wt $\%$ (mean, $\mathrm{SD})$ referred to the initial embolus' weight for each catheter, summarized per clot size $(\geq 5 \mathrm{~mm}, \geq 2 \mathrm{~mm}$, $\geq 1 \mathrm{~mm}), * \mathrm{p}<0.05$, $* * * \mathrm{p}<0.001$

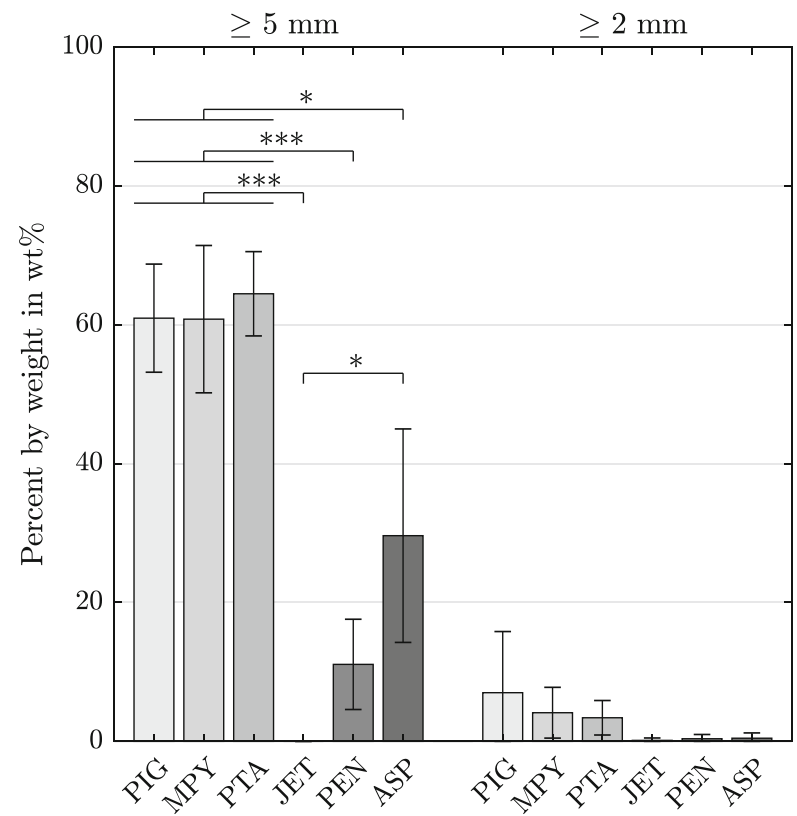

structural integrity of the thrombus, isotonic saline solution would be preferable. However, considering the short time frame of the tests presented, no severe impact on thrombus consistency was expected.

For future studies, blood could be considered as flow medium to include the effect of drug thrombolytic agents in the experimental setup. Additional thrombolytic therapy may be supportive in the case of fragmentation, as the thrombus surface area is increased by mechanical treatment $[14,15]$. Not only blood is crucial, but also the endothelial layer of a natural vascular system and humoral factors, e.g., of an in-vivo intrinsic coagulation system, which makes it difficult to model drug thrombolysis experimentally and invitro.

Moreover, there was no intention of fully covering all available mechanical systems in this study. In clinical settings, large-bore thrombectomy devices with a diameter of 20-F have shown promising results [18]. The intention of our study was to create comparable framework conditions, also with regard to sheath size, which was limited to 8-F, with one exception of 10-F for the ASP catheter. A system with a catheter/sheath size of 20/22-F obviously provides better aspiration properties. However, in the clinical setting, larger system calibers may be associated with higher complication rates such as vascular injury, arrhythmia, and even ventricular fibrillation of a stressed right ventricle [17].

In our experimental setup, clot preparation was aimed at a thrombus with increased consistency and thus increased resistance to aspiration and fragmentation, although this method could not reproduce a partially organized thrombus. So far, no histological examination has been conducted to verify the exact mechanism of consolidation. The important point is that all clots were prepared according to the same protocol, so that comparability between different catheter systems is given. The need for comparability was paramount in our study, so the investigation of very different, old organized or mixed-structured clots, as found in PE in a clinical setting and certainly affecting treatability by catheter systems, was not considered.

Finally, it must be considered that regeneration after PE is a complex process that does not necessarily correlate linearly with pulmonary recanalization by catheter treatment and may be delayed. However, such a regeneration process affects treatment with all catheter systems and unfortunately cannot be reproduced in an in-vitro model.

Despite all these limitations, our setup allowed a comparison of the recanalization properties of the investigated catheters under standardized conditions. The test rig may be also applicable for training and teaching. Procedures can be performed with a direct view onto the transparent vessels or, as an improvement, under sight restriction and with the model placed in a fluoroscopy unit.

\section{Conclusion}

In this study, a comparison of different catheters for mechanical treatment of large-burden PE was performed under standardized in-vitro conditions. It was found that although mechanical thrombectomy worked best with dedicated aspiration devices, embolus fragmentation with simple standard catheters could achieve considerable 
success. If dedicated PE catheters are not available in an emergency, the use of such (slightly modified) standard catheters may be a lifesaving alternative.

Acknowledgements Samples of the aspiration catheters were kindly provided by the companies: JETi ${ }^{\circledR}$ by Walk Vascular LLC., Irvine, CA, USA; Penumbra Indigo ${ }^{\circledR}$ System by Penumbra, Inc., Alameda, CA, USA; Aspirex ${ }^{\circledR}$ by Straub Medical AG, Wangs, Switzerland.

Funding Open Access funding enabled and organized by Projekt DEAL. This study was not supported by any funding.

\section{Declarations}

Conflict of interest Apart from the provision of samples of the aspiration catheters for test purposes (see above), no benefits in any form have been received from a commercial party related directly or indirectly to the subject of this manuscript.

Consent for Publication For this type of study consent for publication is not required.

Ethical Approval Permission to use porcine blood was granted by the responsible veterinary authority in accordance with Article 23 of Regulation (EC) No 1069/2009. No other ethical standards had to be met in the context of this study.

Informed Consent For this type of study informed consent is not required.

Open Access This article is licensed under a Creative Commons Attribution 4.0 International License, which permits use, sharing, adaptation, distribution and reproduction in any medium or format, as long as you give appropriate credit to the original author(s) and the source, provide a link to the Creative Commons licence, and indicate if changes were made. The images or other third party material in this article are included in the article's Creative Commons licence, unless indicated otherwise in a credit line to the material. If material is not included in the article's Creative Commons licence and your intended use is not permitted by statutory regulation or exceeds the permitted use, you will need to obtain permission directly from the copyright holder. To view a copy of this licence, visit http://creativecommons. org/licenses/by/4.0/.

\section{References}

1. Bikdeli B, Monreal M, Jiménez D. Pulmonary embolism in Europe remains a cause of concern despite declining deaths. Lancet Respir Med. 2020;8:222-4. https://doi.org/10.1016/ S2213-2600(19)30360-1.

2. Konstantinides SV, Meyer G, Becattini C, Bueno H, Geersing G-J, Harjola V-P, et al. 2019 ESC Guidelines for the diagnosis and management of acute pulmonary embolism developed in collaboration with the European Respiratory Society (ERS). Eur Heart J. 2020;41:543-603. https://doi.org/10.1093/eurheartj/ ehz405.

3. Barco S, Mahmoudpour SH, Valerio L, Klok FA, Münzel T, Middeldorp S, et al. Trends in mortality related to pulmonary embolism in the European Region, 2000-2015: analysis of vital registration data from the WHO Mortality Database. Lancet Respir Med. 2020;8:277-87. https://doi.org/10.1016/S22132600(19)30354-6.
4. Polat V, Bostancı Gİ. Sudden death due to acute pulmonary embolism in a young woman with COVID-19. J Thromb Thrombolysis. 2020;50:239-41. https://doi.org/10.1007/s11239020-02132-5.

5. Faggiano P, Bonelli A, Paris S, Milesi G, Bisegna S, Bernardi N, et al. Acute pulmonary embolism in COVID-19 disease: Preliminary report on seven patients. Int $\mathrm{J}$ Cardiol. 2020;313:129-31. https://doi.org/10.1016/j.ijcard.2020.04.028.

6. Poissy J, Goutay J, Caplan M, Parmentier E, Duburcq T, Lassalle F, et al. Pulmonary Embolism in Patients With COVID-19: Awareness of an Increased Prevalence. Circulation. 2020;142:184-6. https://doi.org/10.1161/CIRCULATIONAHA. 120.047430 .

7. Kucher N. Catheter embolectomy for acute pulmonary embolism. Chest. 2007;132:657-63. https://doi.org/10.1378/chest.07-0665.

8. Kuo WT. Endovascular Therapy for Acute Pulmonary Embolism. J Vasc Interv Radiol. 2012;23:167-79. https://doi.org/10.1016/j. jvir.2011.10.012.

9. Kuo WT, Gould, Michael K., MS, Louie JD, Rosenberg JK, Sze, Daniel Y., PhD, Hofmann LV. Catheter-directed Therapy for the Treatment of Massive Pulmonary Embolism: Systematic Review and Meta-analysis of Modern Techniques: Catheter-directed Therapy for the Treatment of Massive Pulmonary Embolism: Systematic Review and Meta-analysis of Modern Techniques. J Vasc Interv Radiol. 2009;20(11):1431-40. https://doi.org/10. 1016/j.jvir.2009.08.002.

10. Avgerinos ED, Chaer RA. Catheter-directed interventions for acute pulmonary embolism. J Vasc Surg. 2015;61:559-65. https://doi.org/10.1016/j.jvs.2014.10.036.

11. Nosher JL, Patel A, Jagpal S, Gribbin C, Gendel V. Endovascular treatment of pulmonary embolism: selective review of available techniques. World J Radiol. 2017;9:426-37. https://doi.org/10. 4329/wjr.v9.i12.426.

12. Singh M, Shafi I, Rali P, Panaro J, Lakhter V, Bashir R. Contemporary catheter-based treatment options for management of acute pulmonary embolism. Curr Treat Options Cardio Med. 2021;23:1-18. https://doi.org/10.1007/s11936-021-00920-7.

13. Brady AJB, Crake T, Oakley CM. Percutaneous catheter fragmentation and distal dispersion of proximal pulmonary embolus. Lancet. 1991;338:1186-9. https://doi.org/10.1016/01406736(91)92042-Z.

14. Schmitz-Rode T, Janssens U, Duda SH, Erley CM, Günther RW. Massive pulmonary embolism: percutaneous emergency treatment by pigtail rotation catheter. J Am Coll Cardiol. 2000;36(2):375-80.

15. Schmitz-Rode T, Günther RW, Pfeffer JG, Neuerburg JM, Geuting B, Biesterfeld S. Acute massive pulmonary embolism: use of a rotable catheter for diagnosis and fragmentation therapy. Radiology. 1995;197:157-62.

16. Bayiz H, Dumantepe M, Teymen B, Uyar I. Percutaneous aspiration thrombectomy in treatment of massive pulmonary embolism. Heart Lung Circ 2015. https://doi.org/10.1016/j.hlc.2014. 06.014 .

17. Sista AK, Horowitz JM, Tapson VF, Rosenberg M, Elder MD, Schiro BJ, et al. Indigo aspiration system for treatment of pulmonary embolism: results of the EXTRACT-PE Trial. JACC Cardiovasc Interv. 2021. https://doi.org/10.1016/j.jcin.2020.09. 053.

18. Tu T, Toma C, Tapson VF, Adams C, Jaber WA, Silver M, et al. A prospective, single-arm, multicenter trial of catheter-directed mechanical thrombectomy for intermediate-risk acute pulmonary embolism: the FLARE study. JACC Cardiovasc Interv. 2019. https://doi.org/10.1016/j.jcin.2018.12.022.

19. Schmitz-Rode T, Kilbinger M, Günther RW. Simulated flow pattern in massive pulmonary embolism: significance for 
selective intrapulmonary thrombolysis. CardioVasc Interv Radiol. 1998;21:199-204.

20. Pelletier B, Fritschi A. The artificial heart ReinHeart-system description and current state of the development. Kardiotechnik. 2013:66-70.

21. Schmitz-Rode T, Janssens U, Hanrath P, Günther RW. Fragmentation of massive pulmonary embolism by pigtail rotation catheter: possible complication. Eur Radiol. 2001;11:2047-9. https://doi.org/10.1007/s003300100905.

22. Zana K, Otal P, Fornet B, Forrai G, Chabbert V, Smayra T, et al. In vitro evaluation of a new rotational thrombectomy device: the Straub Rotarex catheter. Cardiovasc Intervent Radiol. 2001. https://doi.org/10.1007/s00270-001-0017-7.

23. Müller-Hülsbeck S, Grimm J, Leidt J, Heller M. In vitro effectiveness of mechanical thrombectomy devices for large vessel diameter and low-pressure fluid dynamic applications. J Vasc Interv Radiol. 2002;13:831-9. https://doi.org/10.1016/S10510443(07)61993-9.

24. Müller-Hülsbeck S, Grimm J, Leidt J, Jahnke T, Heller M. Comparison of in vitro effectiveness of mechanical thrombectomy devices. J Vasc Interv Radiol. 2001;12:1185-91. https://doi.org/10.1016/S1051-0443(07)61678-9.

25. Salazar GM, Faintuch S, Gladstone SR, Lang EV. In vitro analysis of downstream particulates with mechanical thrombectomy devices: comparison of $20-\mathrm{kHz}$ sonothrombolytic and rotating dispersion wire systems. J Vasc Interv Radiol. 2009;20:634-9. https://doi.org/10.1016/j.jvir.2008.12.416.

26. Müller-Hülsbeck S, Brossmann J, Jahnke T, Grimm J, Reuter M, Bewig, Burkhardt M, Heller M. Mechanical thrombectomy of major and massive pulmonary embolism with use of the Amplatz Thrombectomy Device. Investig Radiol. 2001;36:317-22.

27. Knott E, Reul H, Knoch M, Steinseifer U, Rau G. In vitro comparison of aortic heart valve prostheses: Part 1: mechanical valves. J Thorac Cardiovasc Surg. 1988;96:952-61. https://doi. org/10.1016/S0022-5223(19)35164-5.

Publisher's Note Springer Nature remains neutral with regard to jurisdictional claims in published maps and institutional affiliations. 